\title{
Advanced Ion Source Technology for High Resolution and Stable FIB Nanofabrication employing Gallium and new Ion Species.
}

\author{
S. Bauerdick ${ }^{2}$, J. E. Sanabia ${ }^{1}$, P. Mazarov ${ }^{2}$, J. Fridmann ${ }^{1}$, L. Bruchhaus ${ }^{2}$, and R. Jede ${ }^{2}$ \\ 1. Raith America, Inc., 2805 Veterans Memorial Hwy., Ronkonkoma, NY 11779 \\ 2. Raith GmbH, Konrad-Adenauer-Allee 8, 44263 Dortmund, Germany
}

There is a growing interest to use focused ion beam (FIB) systems for nanofabrication and rapid prototyping tasks. We report the status of our continuous effort to advance FIB technology along with an instrumentation platform dedicated to nanofabrication.

FIB systems and combined FIB-SEM microscopes are widely used for sample preparation and various analytical tasks. Instrumentation has seen different developments in recent years in the area of source technology and also in the intended breadth of application space. The Gallium-based LMIS (liquid metal ion source), which has been ubiquitous with -FIB- for many decades, is partly giving way to GFIS (gas field ion source) microscopes, as well as Xenon -plasma- sources. This trend has been mainly driven by ultra-high resolution microscopy and large volume milling for special sample preparation applications, respectively.

Nanofabrication presents different and more demanding requirements for FIB in terms of stability, resolution, and the support of new processing techniques. To advance FIB nanofabrication applications, we report a significant advance in gallium-based LMIS with a stable gun emission design enabling longterm stability without the need for the frequent heating required for conventional gallium ion sources, and producing low drifts in probe current and beam position. Moreover, we report a FIB spot allowing excellent patterning resolution with low collateral damage. A FIB spot is usually not a pure Gaussian distribution, instead exhibiting significant beam tails outside the central spot, which have to be as small as possible for high resolution nanofabrication. In order to measure the beam current distribution we employed a method based on the amorphization of single crystal silicon by Gallium ions, which is a relatively well understood process $[1,2,3]$. For the beam current distribution within the ion spot, a theoretical model consisting of a central large intensity Gaussian, a medium intensity Gaussian and low intensity exponential beam tails is assumed. Compared with conventional LMIS FIB, the results here show a very narrow and large central Gaussian part carrying $>85 \%$ of the total current and very low tails. As the most relevant part for milling is in the dose range of 1 to $10^{-3}$, this technology offers superior performance, especially for nanofabrication.

The type of ion defines the nature of the interaction mechanism with the sample and thus has dramatic consequences the resulting nanostructures. Therefore, we have extended the focused ion beam technology towards the stable delivery of multiple species into a nanometer-scale focused ion beam by employing a liquid metal alloy ion source (LMAIS). A mass separation filter is incorporated into the column to allow for fast and easy switching between different ion species or clusters within minutes $[4,5,6]$. This provides single and multiple charged species of different mass (Figure 1), e.g. Si, Ge and $\mathrm{Au}$, resulting in significantly different interaction mechanisms. We present the capabilities of the multiple-ion instrument including excellent long-term current stability (Figure 1) and sub-10 nm and sub-15 nm beam resolutions for various ions like Be, Si (Figure 2) and Au. The characteristics and applications of different species will be discussed in terms of patterning, functionalization, and imaging. 


\section{References:}

[1] G. Ben Assayag et al., JVST B 11 (1993) 2420.

[2] C. Vieu et al., Nucl. Instrum. Methods B 93 (1994) 439.

[3] S. Tan et al., JVST B 30 (2012) 06F606.

[4] B. R. Appleton et al., Nucl. Instrum. Methods B 272 (2012) 153.

[5] S. Tongay et al., Appl. Phys. Lett. 100 (2012) 073501.

[6] S. Bauerdick et al., JVST B 31 (2013) 06F404.
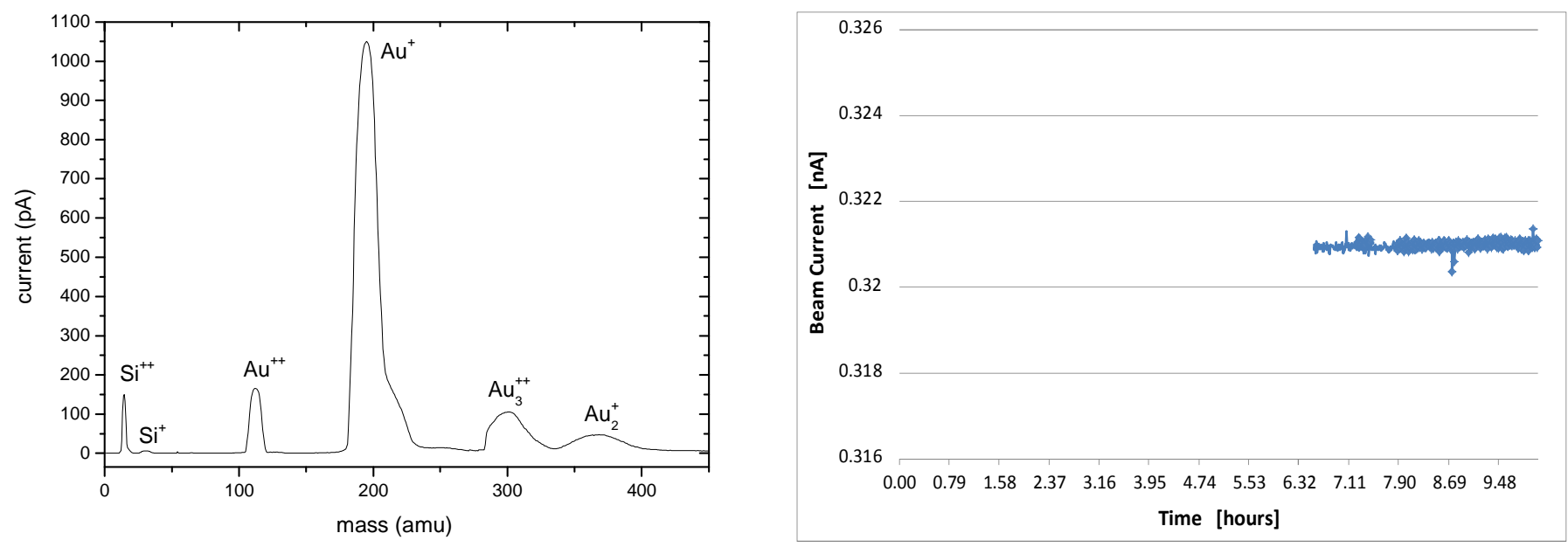

Figure 1. Mass spectrum (showing ions of different charge and mass) and stability measurement over $10 \mathrm{~h}$ of a AuSi Liquid Metal Alloy Ion Source (LMAIS).
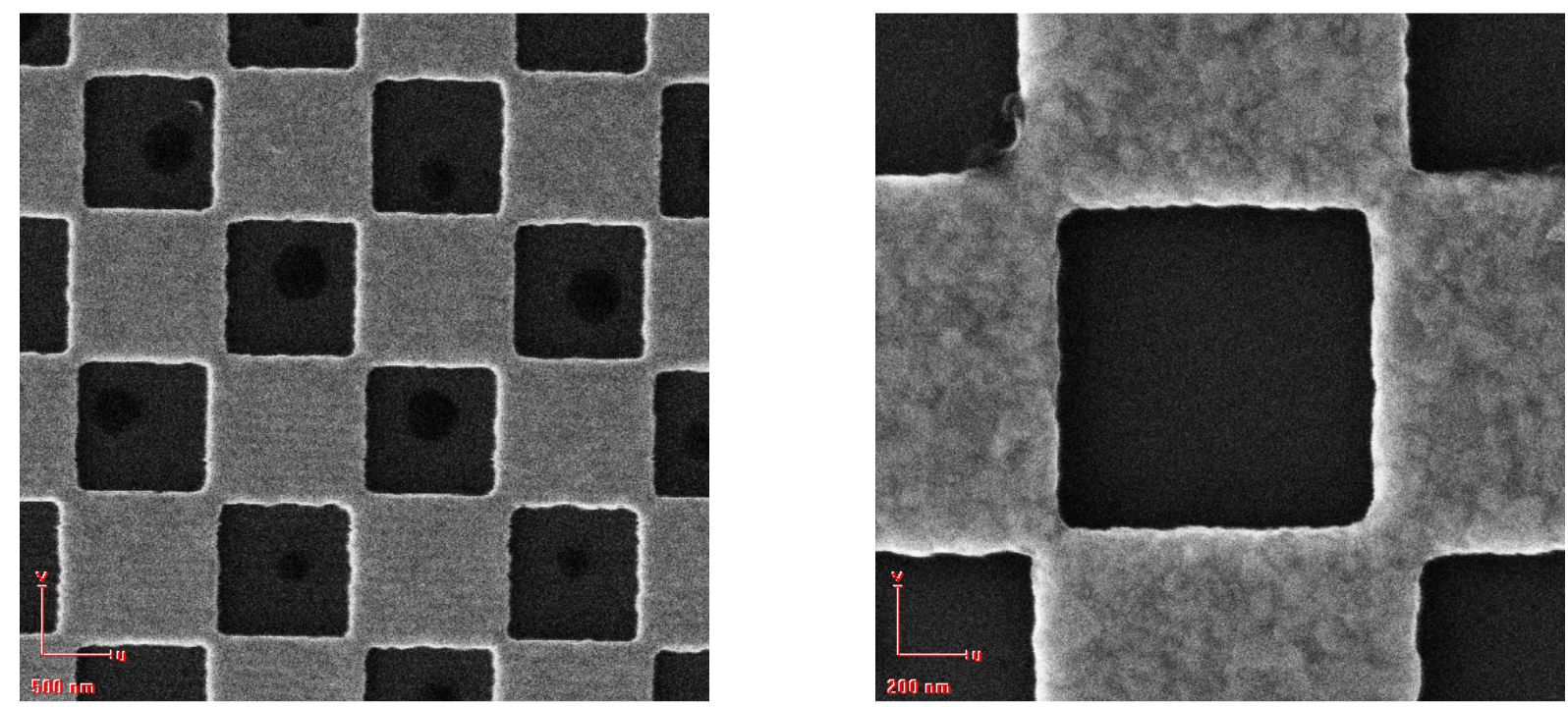

Figure 2. Secondary electron images obtained by scanning a focused $\mathrm{Si}^{++}$(left) or a $\mathrm{Be}^{++}$(right) ion beam over a sample of gold squares $(1 \mu \mathrm{m})$ on silicon. The obtained beam diameters are about 6-8 $\mathrm{nm}$ and 3-4 $\mathrm{nm}$, respectively. 